\title{
Development of vaccines and antivirals for combating viral pandemics
}

\author{
Proactive efforts towards the development of new vaccines and antivirals, and the elimination of bottlenecks in \\ vaccine development, will be essential to containing and eradicating future pandemics.
}

\section{Norbert Pardi and Drew Weissman}

ncreased hygiene, facial protection and social-distancing rules, and the reduction of large gatherings and industrial and commercial activity have helped to 'flatten the case curve' of the coronavirus disease 2019 (COVID-19) pandemic. However, these non-pharmacological measures alone are insufficient in the long term. Successful containment and eradication of pandemic viruses is only possible with prophylactic vaccines. Antiviral drugs (mostly small molecules and neutralizing monoclonal antibodies) can only reduce the morbidity and mortality associated with infection.

There is, therefore, a pressing need for global-preparedness programmes that potentiate our ability to rapidly test existing and newly designed antiviral drugs, and for developing safe, effective, easy-to-produce and reasonably priced vaccines in a timely manner. The unprecedented speed of research and development focused on severe acute respiratory syndrome coronavirus 2 (SARS-CoV-2) in 2020 may serve as a template for mitigating the potentially devastating social and economic consequences of viral pandemics.

The rise of new pathogenic outbreaks in the future is not a matter of 'if', but of 'when'. It is thus imperative that the a priori development of drugs and prophylactic vaccines against viruses, bacteria and other pathogens with pandemic potential is given due consideration. Programmes for global pandemic preparedness are based on experiences from multiple viral epidemics of the past two decades, including those caused by SARS-CoV-1, the H1N1 influenza virus, the chikungunya virus, Middle East respiratory syndrome coronavirus (MERS-CoV), the Ebola virus and the Zika virus.

The high infectivity of pandemic respiratory viruses contrasts with the traditionally slow research and development protocols for new antivirals and vaccines, particularly those based on novel technologies or drug classes. The need for careful safety evaluation and for the expansion of the production capacity of antivirals and vaccines in the setting of a pandemic are additional time-consuming challenges. Current timeframes for drug development, production and distribution are thus not feasible for tackling active pandemic outbreaks. A case in point is an influenza virus vaccine, developed and mass-produced within six months, to combat the pandemic brought by the H1N1 influenza virus (or 'swine flu') a decade ago; this relatively short development timeframe still proved to be too long to influence the outcome of that outbreak ${ }^{1}$. We are experiencing a similar problem today with SARS-CoV-2, for which there is little hope for a mass-produced prophylactic vaccine for human use before 2021 .

Developing novel antiviral agents, including antiviral drugs and vaccines, is financially costly, leaving some to argue that the development of drugs against emerging viruses and other pathogens with pandemic potential is infeasible, especially because viruses can mutate over time and render treatments less effective or even completely ineffective. However, although a proactive approach requires substantial upfront financial investment, it is most appropriate for preparedness. Preparedness involves studying the biology of potentially pandemic pathogens (to understand the mechanism of host cell tropism and to identify small-molecule and vaccine targets, for example), and the pre-emptive development of new drugs or 'prototype' vaccines against a given pathogen or group of pathogens in inter-pandemic periods. Then, in a pandemic scenario, such prototype vaccines (particularly those with genetic formats) can be quickly modified to obtain effective agents. Moreover, because many human pathogens do not replicate well in animals, it can take a long time to generate appropriate animal models to test the protective efficacy of new vaccines, which highlights the importance of laying the groundwork for future research and development before a pandemic. We can certainly learn from past studies on SARS-CoV-1 and MERS-CoV; they represent stepping stones for developing effective vaccines or other drugs for SARS-CoV-2 in a reduced timeframe. In particular, structure-based antigen design is likely to be critical for quickly designing potent vaccines against coronaviruses and against other difficult pathogens (such as the human immunodeficiency virus (HIV), the respiratory syncytial virus and the influenza virus) ${ }^{2}$. Moderna Therapeutics, in collaboration with the Vaccine Research Center at the National Institutes of Health, was able to develop and produce a SARS-CoV-2 mRNA vaccine candidate for human trials in just 42 days $^{3}$, an achievement that might not have been possible without earlier antigen-design studies on MERS-CoV ${ }^{4}$. Additionally, previous work for the development of SARS-CoV-1-specific monoclonal antibodies may become useful as passive immunotherapy against SARS-CoV-2, as both viruses use the same cellular receptor for entry ${ }^{5}$. Clearly, we would likely be in a better position in the battle against SARS-CoV-2 if licensed vaccines against SARS-CoV-1 were available, but no such vaccines have been licensed for human use in the 15 years since the original SARS outbreak $^{6}$. This was a missed opportunity for the development of a potentially cross-protective, ready-to-use vaccine regimen against the novel coronavirus.

Developing antivirals for SARS-CoV-2 Three approaches form the cornerstones of how to confront an outbreak or a new pandemic: community- and behaviour-based actions (isolation, quarantining and social distancing, in particular), small-molecule drugs and monoclonal antibody therapies (for treating and reducing the morbidity and mortality of infected patients), and prophylactic vaccines (for reducing transmission and eventually eradicating the virus from the population). Although community-based actions can be established quickly and are effective in slowing the spread of pathogens, they lose effectiveness as the outbreak progresses owing to their impact 
on local and global economies; in fact, they can lead to serious and long-lasting financial and social consequences ${ }^{7}$.

Pharmaceutical interventions are thus critical for combating pandemics.

Small-molecule drugs can improve the handling of pandemic outbreaks by reducing morbidity and mortality, as exemplified by the antivirals currently used against the hepatitis $B$ virus, the hepatitis $C$ virus, HIV and the herpes simplex virus ${ }^{8}$. These drugs save millions of lives every year. Neutralizing antibodies and small-molecule drugs against SARS-CoV-2 are currently being explored, but one possible shortcut would be to find an already-licensed drug that would also be effective against SARS-CoV-2 and that could be mass-produced; indeed, a large number of drugs have entered clinical trials to assess their potential for repurposing (a regularly updated list of candidates and drug developers is available at https://covid19tracker.milkeninstitute.org).

Chloroquine and hydroxychloroquine, which are licensed antimalarial drugs that are also used to treat patients with rheumatic diseases, have received considerable media attention. But data from multiple clinical trials suggest that these drugs do not provide clinical benefit for patients with COVID-19, and that they can cause adverse effects in these patients ${ }^{9}$. Antivirals developed for other types of RNA viruses - in particular, favipiravir (against the influenza virus) and lopinavir and ritonavir (used in patients with HIV) - have also been studied for their potential activity against SARS-CoV-2. In fact, favipiravir showed some efficacy in patients with mild COVID-19, whereas lopinavir and ritonavir provided no benefit ${ }^{10,11}$. Remdesivir, an adenosine nucleoside analogue developed by Gilead Sciences for treating Ebola virus infections, also entered clinical trials. As remdesivir showed efficacy against SARS-CoV-1, MERS-CoV, SARS-CoV-2 and other coronaviruses in in vitro studies ${ }^{12,13}$, it represents a promising drug candidate for the treatment of SARS-CoV-2 infection in humans. Indeed, a double-blind, randomized, placebo-controlled trial with 1,062 participants showed that, compared with the placebo, remdesivir can shorten the time to recovery of hospitalized adults with COVID-19 and evidence of infection in the lower respiratory tract ${ }^{14}$. On the basis of the positive clinical results, remdesivir was approved for human use ${ }^{15}$ by the United States Food and Drug administration (FDA); however, in November 2020 the World Health Organization recommended against its use in patients with COVID-19 on the basis of insufficient evidence after a review of four randomized controlled trials involving more than 7,000 patients ${ }^{16}$. Still, broad-spectrum antivirals (in particular, nucleoside analogues that target conserved catalytic sites of essential viral enzymes) remain promising drug candidates against SARS-CoV-2.

A number of new drugs not yet approved by the FDA or by the European Medicines Agency are also being studied, including neutralizing monoclonal antibodies. Notably, Regeneron Pharmaceuticals, using a transgenic mouse model that produces human antibodies ${ }^{17}$, has generated neutralizing monoclonal antibodies against SARS-CoV-2 for treatment or as a prophylactic. Clinical evaluation of the safety and efficacy of sarilumab, a human monoclonal antibody against the interleukin-6 receptor, is underway (NCT04315298). Clinical efficacy data for LY-CoV555 (Bamlanivimab), a neutralizing monoclonal antibody developed by Eli Lilly and directed against the spike glycoprotein of SARS-CoV-2, are available ${ }^{18}$. Study participants recently diagnosed with SARS-CoV-2 infection received a single dose of 700, 2,800 or 7,000 $\mathrm{mg}$ of LY-CoV555 or placebo intravenously, and the change of viral load from baseline at day 11 was measured. Surprisingly, only the 2,800 mg dose provided clinical benefit for LY-CoV555-treated patients compared with the group injected with placebo. In November 2020, monoclonal antibodies developed by Regeneron (Casirivimab and Imdevimab) and by Eli Lilly (Bamlanivimab) received emergency use authorization by the FDA ${ }^{19,20}$. Plasma therapy - the transfer of serum from convalescent individuals to acutely ill patients as a passive immunization strategy - is approved by the FDA, yet its efficacy against SARS-CoV-2 infection is still under investigation ${ }^{21}$. Because it proved to be effective in reducing mortality in humans infected with pandemic H1N1 influenza virus ${ }^{22}$, it may be applicable against SARS-CoV-2 as well. Of note, no strong evidence has been provided about the effectiveness of plasma therapy for patients with COVID-19 to date ${ }^{23}$, and further adequate and well-controlled randomized trials will need to investigate the clinical efficacy of this approach.

\section{Developing vaccines for SARS-CoV-2}

Although SARS-CoV-2 is a novel human virus, we can take advantage of existing knowledge stemming from research into vaccines for the related SARS-CoV-1 and MERS-CoV. Potential SARS-CoV-2 vaccine targets have been identified (Fig. 1a), and the scientific community has experience with the design of coronavirus antigens ${ }^{4}$. Importantly, the genetic sequence of the virus has been publicly available since January 2020, and animal models that were developed for the evaluation of SARS-CoV-1 vaccines may also be useful for assessing SARS-CoV-2 vaccine candidates ${ }^{24}$. Many pharmaceutical companies and academic research institutions have since launched SARS-CoV-2 vaccine programmes using both conventional and genetic or viral vector-based platforms ${ }^{25}$.

SARS-CoV-2 vaccines can be divided into three general groups: nucleic-acid-based, protein-based (including the use of inactivated viruses and virus-like particles), and live-vector-based. Table 1 provides a summary of the leading SARS-CoV-2 clinical vaccine candidates supported by Operation Warp Speed in the United States.

Among the nucleic-acid formulations, mRNA-based vaccines are a promising strategy and include multiple vaccine candidates against COVID-19 (refs. ${ }^{26-28}$ ). One such strategy employs a nucleosidemodified mRNA-lipid nanoparticle (mRNA-LNP) vaccine encoding either the full-length SARS-CoV-2 spike glycoprotein (with a mutated furin cleavage site) or the receptor-binding domain of the spike protein as a monomer ${ }^{28}$. In mice, both vaccines induced robust type- $1 \mathrm{CD}^{+}$and $\mathrm{CD}^{+} \mathrm{T}$-cell responses in the spleen and lungs after administration of a single $30 \mu \mathrm{g}$ dose. Moreover, the vaccines elicited strong and long-lived plasma-cell and memory-B-cell responses that were associated with the production of antibodies with potent anti-SARS-CoV-2 neutralization activity. Moderna's mRNA-1273 vaccine, which contained nucleoside-modified mRNA-LNPs encoding the spike protein of SARS-CoV-2 stabilized in the pre-fusion state $^{26,27}$, also elicited robust T-helper1-dominant $\mathrm{CD}^{+}$and $\mathrm{CD}^{+}{ }^{+} \mathrm{T}$-cell responses after two immunizations with only $1 \mu \mathrm{g}$ of mRNA-LNPs in mice ${ }^{26}$. Importantly, the vaccine induced strong neutralizing antibody responses and protection from viral replication in the lungs of SARS-CoV2-challenged mice. When tested in rhesus macaques, two immunizations with 10 or $100 \mu \mathrm{g}$ of mRNA-1273 induced T-helper-1-biased CD4 ${ }^{+} \mathrm{T}$-cell responses and neutralizing antibody responses in a dose-dependent manner ${ }^{27}$. Interestingly, no $\mathrm{CD}^{+} \mathrm{T}$-cell responses could be measured in vaccinated non-human primates, but the mRNA-1273 vaccine could induce a high level of protection (particularly in the high-dose group) from viral replication in the upper and lower respiratory tracts.

There are a few clinical reports of the safety and efficacy of mRNA-based SARS-CoV-2 vaccine candidates tested in a small number of people ${ }^{29,30}$. In a study 
a

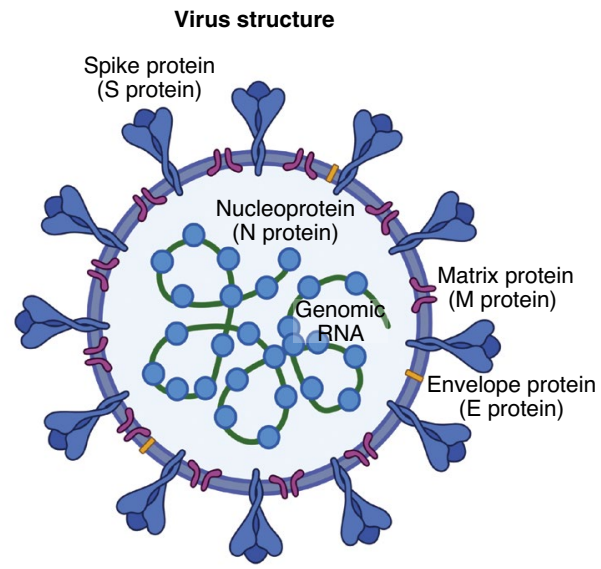

Vaccine targets
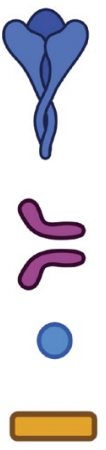

Vaccine types

RNA vaccine

DNA vaccine

Recombinant protein vaccine

Viral-vector-based vaccine

Live-attenuated-virus vaccine

Inactivated-virus vaccine b
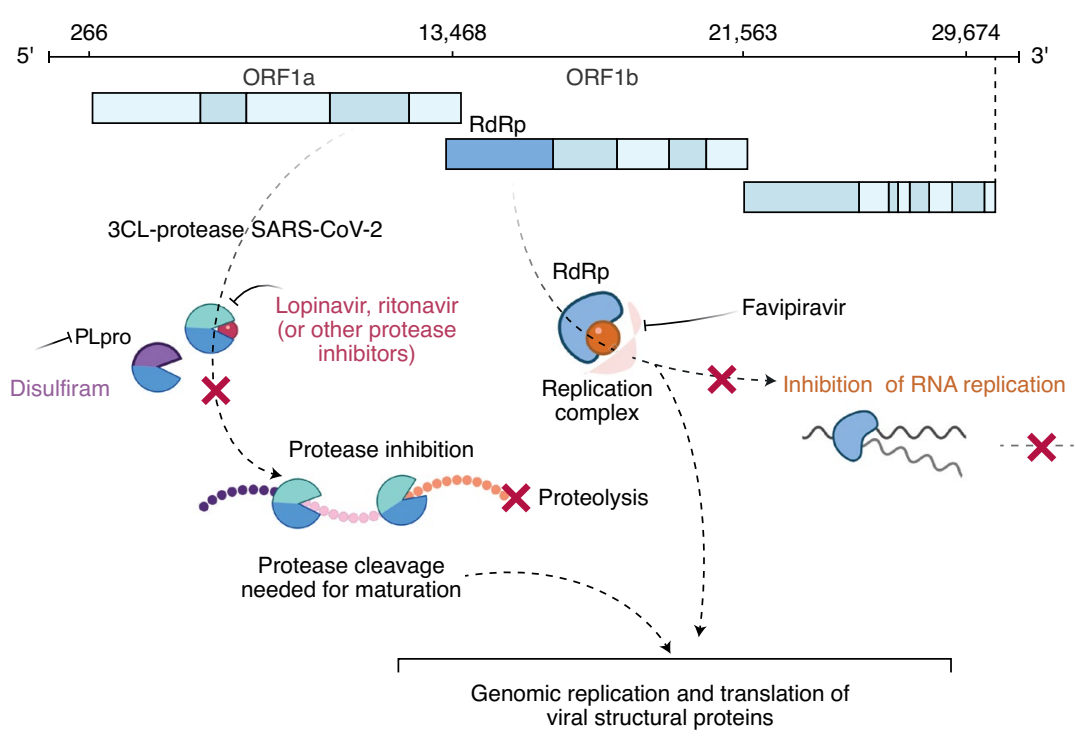

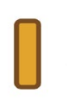

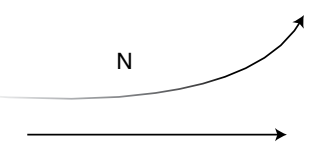

$S, M$ and $E$ at $E R$ membrane
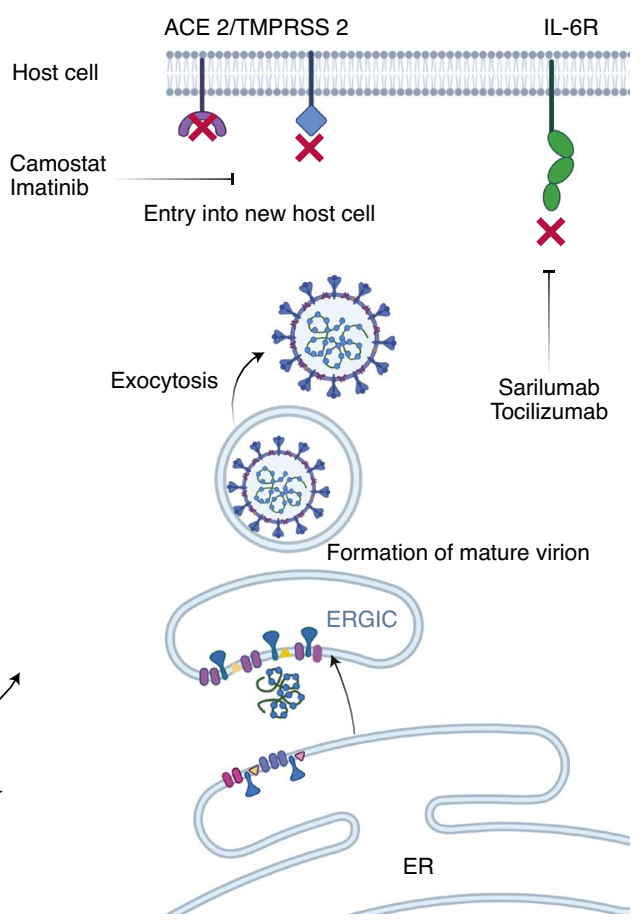

Fig. 1 | Prophylactic and therapeutic strategies against SARS-CoV-2. a, Vaccine targets and vaccine platforms against SARS-CoV-2. b, Examples of viral targets and of potential antiviral drugs against SARS-CoV-2. ORF, open reading frame; RdRp, RNA-dependent RNA polymerase; PLpro, papain-like protease; ACE 2, angiotensin-converting enzyme 2; TMPRSS 2, transmembrane protease, serine 2; ER, endoplasmic reticulum; ERGIC, endoplasmic-reticulum-Golgi intermediate compartment; IL-6R, interleukin-6 receptor. Image courtesy of Mohamad-Gabriel Alameh.

of Moderna's mRNA-1273 vaccine ${ }^{29}, 45$ (mainly white) volunteers aged 18-55 received two intramuscular immunizations of 25,100 or $250 \mu \mathrm{g}$ mRNA-LNPs 4 weeks apart. The results are promising, as the median magnitude of neutralizing antibody responses measured in the vaccinees was in the upper quartile of values in convalescent serum samples, and antibody titres correlated with the vaccine dose. In general, the vaccine was well tolerated, although over half of the participants receiving the
100 and $250 \mu \mathrm{g}$ doses reported fever and other adverse events (including fatigue, chills, headache, or pain at the injection site) after administration of the second dose. Vaccine evaluation in a phase 3 clinical trial (NCT04470427) will be necessary to confirm these results and to provide more detailed comparative data on vaccine safety and efficacy in different age and ethnic groups. The nucleoside-modified mRNA-LNP vaccine developed by Pfizer/ BioNTech $^{30}$, which encodes a trimeric form of the receptor-binding domain of the SARS-CoV-2 spike protein, has also been tested in human volunteers. Forty-five (mainly white) individuals aged 19-54 received two intramuscular immunizations of 10 or $30 \mu \mathrm{g}$ mRNA-LNPs 3 weeks apart, or a single dose of $100 \mu \mathrm{g}$ mRNA-LNPs. The safety and immunogenicity results were similar to those of the Moderna vaccine, with dose-dependent neutralizing antibody titres and mild or moderate adverse events. The booster immunizations elicited 
Table 1 | Leading SARS-CoV-2 vaccine candidates supported by Operation Warp Speed in the United States

\begin{tabular}{|c|c|c|c|}
\hline Vaccine & Technology platform & Developers & Development status and clinical trial number \\
\hline mRNA-1273 (ref. ${ }^{29}$ ) & mRNA & Moderna Therapeutics and NIAID & $\begin{array}{l}\text { Phase } 3 \\
\text { NCT0 } 4470427\end{array}$ \\
\hline BNT162b2 (ref. ${ }^{30}$ ) & mRNA & $\begin{array}{l}\text { BioNTech RNA Pharmaceuticals and } \\
\text { Pfizer }\end{array}$ & $\begin{array}{l}\text { Phase } 3 \\
\text { NCT0 } 4368728\end{array}$ \\
\hline AZD1222 (ref. ${ }^{33}$ ) & Adenovirus vaccine & $\begin{array}{l}\text { University of Oxford and } \\
\text { AstraZeneca }\end{array}$ & $\begin{array}{l}\text { Phase } 3 \\
\text { NCT0 } 4516746\end{array}$ \\
\hline Ad26.COV2-S & Adenovirus vaccine & Johnson \& Johnson & $\begin{array}{l}\text { Phase } 3 \\
\text { NCT0 } 4505722\end{array}$ \\
\hline- & $\begin{array}{l}\text { Vesicular stomatitis virus- and } \\
\text { measles-based }\end{array}$ & Merck Sharp and Dohme Corp. & $\begin{array}{l}\text { Phase } 1 / 2 \\
\text { NCT0 } 4498247\end{array}$ \\
\hline
\end{tabular}

significantly stronger neutralizing antibody responses. Of note, using lower vaccine doses $(10-30 \mu \mathrm{g})$ to achieve protection from viral infection can be a critical advantage when millions or even billions of doses of a vaccine need to be rapidly manufactured. Similar to the Moderna vaccine, a larger study (NCT04368728) will shed light on the potential differences in safety and efficacy between different ethnicities and age groups. In November 2020, Moderna and Pfizer/ BioNTech announced final-efficacy ${ }^{31,32}$ results, from the phase 3 trials of the mRNA-1273 and BNT162b2 vaccines; the headline $94-95 \%$ efficacies and the absence of serious safety concerns are highly promising.

Preclinical and clinical data are also already available for other vaccine platforms. One of the SARS-CoV-2 vaccine candidates at the most advanced development stage is based on a replication-deficient simian adenovirus vector, ChAdOx1, which contains the full-length spike protein with a tissue-plasminogen-activator leader sequence ${ }^{33}$. A single intramuscular immunization of $5 \times 10^{10}$ viral particles in healthy adults induced neutralizing antibody responses in $100 \%$ of the participants, and a subsequent immunization further increased the neutralizing antibody titres to levels comparable to those measured in convalescent plasma samples. The vaccine was well tolerated, as only mild and moderate adverse events were observed after vaccine administration. Another trial of human volunteers receiving a single intramuscular immunization of a non-replicative adenovirus type 5 (Ad5) vaccine containing the SARS-CoV-2 spike protein $^{34}$ showed that immunization induced cellular and humoral immune responses in most immunized individuals; however, at least one adverse reaction was reported for more than $75 \%$ of the participants within 7 days post-immunization. Three-quarters of the highest-dose group had a fourfold increase in neutralizing antibodies, but no comparison with titres from convalescent patients was performed. Of note, most humans have pre-existing neutralizing antibodies against several adenovirus serotypes, including Ad5, which can negatively affect the performance of such vaccines ${ }^{35}$.

Inovio's DNA vaccine (INO-4800), which targets the spike protein of SARS-CoV-2, was also shown to be immunogenic in mice and guinea pigs, although the protective efficacy of the vaccine has not yet been evaluated ${ }^{36}$. A series of SARS-CoV-2 spike-protein-based DNA vaccines (both soluble and transmembrane) tested in rhesus macaques ${ }^{37}$ induced neutralizing antibody responses and variable levels of protection from SARS-CoV-2 infection following two intramuscular immunizations (on week 0 and week 3 ) with $5 \mathrm{mg}$ of the vaccine. Others have evaluated an alum-adjuvanted purified inactivated SARS-CoV-2 vaccine (PiCoVacc) in mice, rats and rhesus macaques $^{38}$; the vaccine was immunogenic in mice and rats after two immunizations, and elicited protective immune responses from SARS-CoV-2 infection after three immunizations (on days 0, 7 and 14) with 3 or $6 \mu \mathrm{g}$ doses in non-human primates.

On one hand, the development of conventional vaccines (such as those based on recombinant protein subunits and on inactivated or live-attenuated viruses) has a critical advantage over novel vaccine formats: researchers have a deep pool of relevant experience to draw from (in particular, safety data), and similar vaccine formats for humans are already in use. This previous experience will likely speed up the licensing of SARS-CoV-2 vaccines that use conventional vaccine platforms, and their mass production will benefit from infrastructure that is readily available. Nevertheless, as with any new medicine, their initial development still requires extensive investigation. On the other hand, genetic vaccines have already showed high efficacy in preclinical models, and offer the advantages of flexible and very fast antigen design, and of rapid production (once sufficient manufacturing capacity is available) $)^{39}$. Some of these advantages are exemplified by the unprecedented speed of the generation of a SARS-CoV-2 mRNA vaccine for human testing by Moderna Therapeutics.

The concerted effort being poured into SARS-CoV-2 vaccine development, with multiple platforms being explored in varied forms, should ensure that there will be at least one viable vaccine licensed for SARS-CoV-2. At this point, the bottlenecks are the time-consuming nature of clinical trials (which are likely to span many months in the best-case scenario, particularly if the administration of a single vaccine dose does not induce durable protective immune responses), and the mass production and distribution of the vaccines (which could take months or even years if billions of vaccine doses must be produced and administered worldwide).

\section{Bottlenecks in vaccine development}

Vaccine development is a complex endeavour. It involves multiple phases, including an initial design stage, preclinical studies, phases 1-3 of clinical trial testing, approval for human use, and post-marketing studies. Each development phase faces its challenges and adds to the overall length of the process. Early preclinical studies alone, which aim to establish the safety and efficacy of the vaccine platform in animal models in the context of the pathogen of interest, can take decades to complete; for example, preclinical studies for the development of a HIV vaccine have been ongoing for almost 40 years. And that is even before beginning human testing, which (in the United States) involves formal toxicity trials in animals, followed by an Investigational New Drug submission to the FDA, phase 1 tests in 
small groups of people who receive the vaccine (to test dosing and safety), phase 2 clinical studies that expand testing to additional patients to assess efficacy and adverse effects, and phase 3 trials in which the vaccine is given to thousands of people in a placebo-controlled, double-blind protocol for the evaluation of efficacy and safety. The duration of phase 3 testing alone depends in part on the incidence of infection and on the characteristics of the disease (acute infection versus chronic infection).

Altogether, the sheer length of this formal process is a major roadblock in the development of a safe and efficacious vaccine to tackle the spread of a pandemic virus. In the context of the COVID-19 pandemic, researchers, governments, pharmaceutical companies and regulatory bodies are coordinating an unprecedented overhaul of the vaccine development process by condensing - rather than eliminating - steps in the process, without compromising safety. Preclinical testing can be condensed into a much shorter time period when exploring vaccine strategies developed for related pathogens. The speed at which the three phases of clinical testing can be completed depends on many factors, including the disease being targeted. One of the most important factors in phase 3 trials is the incidence of the disease in the target population, which determines the size of the study and the duration of follow-up needed to obtain compelling data. Once approved - or during the approval process itself the protocols for developing the necessary manufacturing scale according to good manufacturing practice (GMP) standards are a critical concern. Finally, comprehensive distribution of the vaccine to the population, and the education and legislation required to encourage the majority of people to get the vaccine, are extremely important considerations. Overall, it is believed that at least 8-12 months will be needed to evaluate novel SARS-CoV- 2 vaccine candidates and to produce sufficient doses for people at high risk (such as healthcare workers and individuals with chronic diseases). Vaccine production for the rest of the world's population and comprehensive vaccine distribution and administration will likely take at least an additional 6-8 months.

Even putting aside the lengthiness of formal vaccine development programmes, bottlenecks and delays may occur at any step of the process. Most vaccines do not move out of preclinical testing, owing to a lack of efficacy or to toxicity. And multiple factors - toxicity, adverse events (including unexpected incidents), lack of efficacy, poor magnitude or short durability of the protective response, projected cost and the inability to effectively implement GMP manufacturing - determine whether a vaccine will continue its development and be submitted for approval. Individual adverse effects identified in human volunteers during clinical trials may lead to the trial being placed on hold (allowing researchers to investigate the adverse effect and assess whether it compromises vaccine safety), as has occurred with several of the SARS-CoV-2 vaccine formulations that are currently in phase 3 trials. Vaccine developers can address many bottlenecks by altering the adjuvant or other components to reduce toxicity, developing new production methods to reduce cost, and adjusting the dose, the location of delivery and the timing and number of immunizations to increase efficacy.

\section{Outlook}

The SARS-CoV-2 pandemic is an extraordinary health emergency that has profoundly altered lives around the globe. It requires unprecedented and globally coordinated steps. The virus is likely to stay with us until a prophylactic vaccine is developed, produced and administered worldwide, which is not expected to happen until 2021. Besides social isolation, our current best chance to reduce SARS-CoV2-related morbidity and mortality is to find and apply a licensed drug (such as a small molecule, Fig. 1b) with at least partial effectiveness against SARS-CoV-2 infection. Owing to tremendous research efforts in this area, the identification of an effective antiviral drug that can be widely distributed is a concrete possibility. But the typical development programme and careful evaluation of novel antivirals and vaccines take years - a timeframe that falls short in the context of an ongoing pandemic.

To avoid or mitigate the consequences of future pandemics, policymakers (such as governments and the World Health Organization) and the pharmaceutical industry need to recognize that sustained and well-organized global pandemic preparedness is critical. Activities should include monitoring the natural reservoirs of pathogens with pandemic potential, improving communication between different national and international centres of disease control, the development and licensure of novel (preferably broad-spectrum) antivirals and antibacterials, the development of 'prototype' vaccines that can quickly be adjusted to a pandemic pathogen and be produced and distributed at large scale, and the generation of appropriate animal models for testing the safety and protective efficacy of novel vaccine candidates. These measures require substantial investment from national governments, but these expenses more than make up for the financial (and human) costs of handling a pandemic, as is patent from the heavy effects of lockdowns on global (and personal) economies during the first wave of SARS-CoV-2 outbreaks. Resources should also be poured into communicating with the public frankly and effectively about vaccines, and about the science of pandemics and the measures to slow transmission. Effective science communication is particularly critical when addressing anti-vaccine (and anti-science) activities in the general population, as they can undermine the bench-to-bedside translation of successful research.

Norbert Pardi ${ }^{\bowtie}$ and Drew Weissman (D) $₫$ Department of Medicine, University of Pennsylvania, Philadelphia, PA, USA.

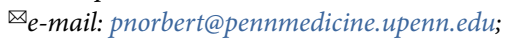
dreww@pennmedicine.upenn.edu

Published online: 8 December 2020 https://doi.org/10.1038/s41551-020-00658-w

References

1. Krammer, F. \& Palese, P. Nat. Rev. Drug Discov. 14, 167-182 (2015).

2. Graham, B. S., Gilman, M. S. A. \& McLellan, J. S. Annu. Rev. Med. 70, 91-104 (2019)

3. Moderna ships mRNA vaccine against novel coronavirus (mRNA-1273) for phase 1 study. Moderna, Inc. https://investors. modernatx.com/news-releases/news-release-details/modernaships-mrna-vaccine-against-novel-coronavirus-mrna-1273 (2020).

4. Pallesen, J. et al. Proc. Natl Acad. Sci. USA 114, E7348-E7357 (2017).

5. Hoffmann, M. et al. Cell 181, 271-280 (2020).

6. Tse, L. V., Meganck, R. M., Graham, R. L. \& Baric, R. S. Front. Microbiol. 11, 658 (2020).

7. Duan, H., Wang, S. \& Yang, C. Nature 578, 515 (2020).

8. De Clercq, E. \& Li, G. Clin. Microbiol. Rev. 29, 695-747 (2016).

9. Boulware, D. R. et al. N. Engl. J. Med. 383, 517-525 (2020).

10. Cao, B. et al. N. Engl. J. Med. 382, 1787-1799 (2020).

11. Dong, L., Hu, S. \& Gao, J. Drug. Discov. Ther. 14, 58-60 (2020).

12. Gordon, C. J. et al. J. Biol. Chem. 295, 6785-6797 (2000).

13. Sheahan, T. P. et al. Sci. Transl. Med. 396, eaal3653 (2017)

14. Beigel, J. H. et al. N. Engl. J. Med. 383, 1813-1826 (2020).

15. FDA approves first treatment for COVID-19. United States Food and Drug Administration https://www.fda.gov/news-events/ press-announcements/fda-approves-first-treatmentcovid-19 (2020).

16. WHO recommends against the use of remdesivir in COVID-19 patients. World Health Organization https://www.who.int/news$\mathrm{room} /$ feature-stories/detail/who-recommends-against-the-useof-remdesivir-in-covid-19-patients (2020).

17. Macdonald, L. E. et al. Proc. Natl Acad. Sci. USA 117, 292-299 (2020).

18. Chen, P. et al. N. Engl. J. Med. https://doi.org/10.1056/ NEJMoa2029849 (2020).

19. FDA authorizes monoclonal antibodies for treatment of COVID-19. United States Food and Drug Administration https://www.fda.gov/news-events/press-announcements/ coronavirus-covid-19-update-fda-authorizes-monoclonal-ant ibodies-treatment-covid-19 (2020).

20. FDA authorizes monoclonal antibody for treatment of COVID-19. United States Food and Drug Administration https://www.fda.gov/news-events/press-announcements/ coronavirus-covid-19-update-fda-authorizes-monoclonal-ant ibody-treatment-covid-19 (2020).

21. Zhang, B. et al. Chest 158, E9-E13 (2020).

22. Hung, I. F. et al. Clin. Infect. Dis. 52, 447-456 (2011).

23. Li, L. et al. JAMA 324, 460-470 (2020).

24. Gretebeck, L. M. \& Subbarao, K. Curr. Opin. Virol. 13, 123-129 (2015).

25. Amanat, F. \& Krammer, F. Immunity 52, 583-589 (2020). 
26. Corbett, K. S. et al. Nature 586, 567-571 (2020).

27. Corbett, K. S. et al. N. Engl. J. Med. 383, 1544-1555 (2020).

28. Laczko, D. et al. Immunity 53, 724-732 (2020).

29. Jackson, L. A. et al. N. Engl. J. Med. https://doi.org/10.1056 NEJMoa2022483 (2020)

30. Mulligan, M. J. et al. Nature 586, 589-593 (2020).

31. Moderna announces primary efficacy analysis in phase $3 \mathrm{COVE}$ study for its COVID-19 vaccine candidate and filing today with U.S. FDA for emergency use authorization. Moderna Therapeutics https://investors.modernatx.com/news-releases/news-releasedetails/moderna-announces-primary-efficacy-analysis-phase3-cove-study (2020).

32. Pfizer and BioNTech conclude phase 3 study of COVID-19 vaccine candidate, meeting all primary efficacy endopoints. Pfizer https:// www.pfizer.com/news/press-release/press-release-detail/pfizerand-biontech-conclude-phase-3-study-covid-19-vaccine (2020).
33. Folegatti, P. M. et al. Lancet 396, 467-478 (2020). 34. Zhu, F. C. et al. Lancet 395, 1845-1854 (2020).

35. Fausther-Bovendo, H. \& Kobinger, G. P. Hum. Vacc. Immunother. 10, 2875-2884 (2014)

36. Smith, T. R. F. et al. Nat. Commun. 11, 2601 (2020)

37. Yu, J. et al. Science 6505, 806-811 (2020).

38. Gao, Q. et al. Science 6499, 77-81 (2020).

39. Pardi, N., Hogan, M. J., Porter, F. W. \& Weissman, D. Nat. Rev. Drug Discov. 17, 261-279 (2018)

Acknowledgements

We thank M. J. Hogan and K. Walsh for a critical reading of the manuscript. N.P. is supported by the National Institute of Allergy and Infectious Diseases (NIAID grant nos. AI146101 and AI153064) and the Global
Health Innovative Technology Fund (GHIT). D.W. is supported by the NIAID (grant nos. R01-AI124429, BAA2018, AI124429, AI142596, 1U19AI135902 and UM1-AI-144371) and BioNTech RNA Pharmaceuticals.

\section{Competing interests}

The authors are named on patents (20170327842, $10,232,055,20180303925)$ that describe the use of nucleoside-modified mRNA as a platform to deliver therapeutic proteins and vaccines. These competing interests have been fully disclosed to the University of Pennsylvania, and an approved plan for managing any potential conflicts arising from the licensing of the patents is in place. 